\title{
Severe Gender Identity Disorder in a Patient with Salt Losing Congenital Adrenal Hyperplasia \\ V Singh $^{1}$, S Soman ${ }^{1}$, C Cave $^{2}$
}

\section{INTRODUCTION}

We highlight the case of a female patient who underwent masculinization both biologically as well as psycho-socially as a result of sub-optimal management of salt losing $21 \alpha$-OHlyase deficiency form of congenital adrenal hyperplasia (CAH.)

Keywords: Congenital adrenal hyperplasia(CAH), gender identity disorder, testosterone, female

From: ${ }^{1}$ Child Health Unit, Faculty of Clinical Medical Science, University of the West Indies, Eric Williams Medical Sciences Complex, Trinidad and Tobago. ${ }^{2}$ Pre-Academic Foundation Programme, Oxford University NHS Trust, United Kingdom (BmBCH - Oxon)

Correspondence: Dr V Singh, Faculty of Clinical Medical Science, University of the West Indies, Eric Williams Medical Sciences Complex, Trinidad and Tobago.

Fax: +1868 662 9596; e-mail: virendra.singh@sta.uwi.edu 


\section{CASE REPORT}

A 19 year old female, an Islamic girl was diagnosed with salt-wasting CAH soon after birth. The diagnosis was made clinically at birth and followed that of an elder brother who underwent a salt-wasting crisis. Her brother died during a salt-wasting crisis in adolescence both as a result of adherence as well as unavailability of medications. The patient's treatment consisted of steroid supplementation with hydrocortisone and fludrocortisone once daily. She experienced progressive and marked postnatal virilisation associated with adult male testosterone levels repeatedly documented from the age of 5 years old (levels ranging from $5.0-9.5$ adult male range $4.56-28.2 \mathrm{nmol} / \mathrm{l}$; adult female range 0.198 to 2.67.) She subsequently developed a masculine build combined with marked cliteromegaly (reasonably male sized, Pradder G3) prior to the onset of puberty. From the age of twelve, she reported being attracted to females and confusion about her gender. By the age of 14 she repeatedly voiced a desire to have gender reassignment. Her mother, having witnessed her being treated similar to a male child (“.. are you sure that is not a boy?” would be a frequent question jested in the home circles as she wore a face covering hijab) is in full support of alleviating her daughter's psychological discomfort and supports corrective surgery and gender reassignment.

Our patient was advised to try maintaining two different lifestyles in an effort to determine which she would be more comfortable with. The first consisted of having relationships with women while identifying with (and being identified by her partners as having) a female gender identity; essentially engaging in same gender relationships. The second was to engage in relationships where she identifies herself and is identified by her partners within a male role. She attempted two relationships subsequent to this discussion. On both occasions she adopted the second scenario of being identified as the male partner within a cross gender relationship. Both relationships failed because she continued to see herself 
within a male role whilst her partners on both occasions tried to convince her not to undergo sex reassignment surgery. These partners preferred her within a female role. That gender identity she simply would not adopt. She has to this time 2 years later refused to engage in same gender relationships as she cannot perceive herself as female at all.

Her desire to have simple SRS, would require a minimum mastectomy and adult male testosterone supplementation.

\section{DISCUSSION}

Physiologically, androgens have a masculinising effect on the foetal brain.(1). Many genetic females raised in a prenatal environment of abnormally high testosterone levels, as typified in untreated 21-Hydroxylase $\mathrm{CAH}$, have masculinised behaviour and other attributes(1) due to the effect of testosterone on the brain and body, as seen in the subject of this case report. Nordenstrom et al (2003) conducted a study wherein the gender-based activities of 26 2-10 yr old CAH girls were studied and compared with 26 unaffected girls matched for age(2). All of the girls were diagnosed and treated as neonates, so that any masculinisation was mainly due to prenatal androgen exposure. They showed that $\mathrm{CAH}$ girls were more interested in masculine toys (bus, construction toys for example), male playmates and masculine careers (soldier, pilot) than female controls. The study also established a relationship between disease severity and masculinity, with those girls having greater levels and longer androgen exposure showing greater degrees of masculine behaviour. Several other studies have shown that CAH girls have more in common with males than females such as play activities(3,4,5,6,)aggression(7), spatial ability(8). 


\section{Gender identity and the CAH female}

Gender assignment occurs at or before the birth of a baby, usually based on genital appearances prenatally or at birth. Gender identity refers to the gender with which an individual identifies(9). The act of gender assignment comes with certain expectations; for example that the inward genitalia would match the outward and that the appropriate gender identity will follow(10). However, in some cases, the gender identity may not necessarily match gender assignment or rearing, as seen in the subject of this report who is genetically female, reared as a female but perceives herself as male. Gender identity is a complex result of the interaction of several factors, including biological influences such as pre and post natal hormonal levels, gene regulation,(11) and social factors such as gender messages from family, institutions and the media (12). Our patient not only had masculinisation of the brain, but her masculine features led others to treat her like a male, socially influencing her gender identity. Most patients with Gender Identify Disorders do not actually have biological or hormonal abnormalities.

Even though this $\mathrm{CAH}$ female displays extreme gender dysphoria, studies have shown that up to $95 \%$ of females with $\mathrm{CAH}$ have no gender dysphoria and identify themselves as female $(13,14,15)$. However, a larger proportion of CAH females are homosexual / bisexual when compared with the rest of the population(16). The use of once daily hydrocortisone (half life of 6-8 hours) was biologically insufficient to effectively suppress ACTH production. Once ACTH driven, her adrenals in an attempt to synthesise cortisol produces excessive testotserone. This resulted in heightened ACTH-driven androgen production during the daily periods of non-suppression of ACTH. Consequently, our patient already antenatally conditioned continued to undergo significant post-natal masculinisation. Studies also highlight that a greater percentage of $\mathrm{CAH}$ females have Gender Identity Disorder when compared with the general population(13). Of those identifying themselves as females, a 
sizeable number have difficulty establishing intimate relationships and have lower fertility than control females $(14,15)$.

\section{Lessons to be learnt}

To obtain optimal outcomes antenatal treatment with adrenal suppressing doses of steroids are necessary if couples are at known risk. Postnatal therapy ought not just to replace adrenal function but aim to at all times suppress the ACTH driven steroidogenesis attempted by the adrenals. This process should be sustained throughout the 24 hours of a day as any breakthrough adrenal functioning serves to create excessive amounts of testosterone which masculinises. Appropriate doses and dose intervals must therefore be employed. The condition is very heterogenous due to a variety of defects in emzyme activity and therefore patient care should be well individualised.

Monitoring of the adequacy of adrenal suppression can be done by monitoring the patient clinically for lack of androgen effects, growth rates, early am testosterone and ACTH levels, 17 OH-P levels during the day, blood pressure, electrolytes and plasma renin activity.

\section{CONCLUSION}

Our patient with $\mathrm{CAH}$ experienced antenatal masculainisation of her brain. Her sub-optimal adrenal suppression resulted in further postnatal masculinisation biologically, physically and psychosocially resulting in a persistent desire for gender reassignment. 


\section{REFERENCES}

1. Berenbaum, S. A., Baxter, L., Seidenberg, M., \& Herman, B. Role of hippocampus in sex differences in verbal memory: Memory outcome following left anterior temporal lobectomy. Neuropsychology 1997;11: 585-591.

2. Nordenstro“m, A., Larsson, A., Servin, A.,Bohlin, G. Prenatal Androgens and Gender-Typed Behavior: A Study of Girls With Mild and Severe Forms of Congenital Adrenal Hyperplasia. Dev Psychol 2003, Vol. 39, No. 3, 440-450.

3. Cole D \& La Voie J. Fantasy play and related cognitive development in 2- to 6-yearolds. Dev Psychol 1985; 21: 233-240.

4. Hines M, \& Kaufman F. Androgens and the development of human sex-typical behavior: Rough-and-tumble play and sex of preferred playmates in children with congenital adrenal hyperplasia (CAH). Child Dev 1994; 65: 1042-1053.

5. Berenbaum S \& Hines M. Early androgens are related to childhood sex-stereotyped toy preferences. Psychol Sci 1992; 3: 203-206.

6. Berenbaum S \& Snyder E. Early hormonal influences on childhood sex-stereotyped activity and playmate preferences: Implications for the development of sexual orientation. Dev Psychol 1995; 3: 31-42.

7. Berenbaum S \& Resnick S. Early androgen effects on aggression in children and adults with congenital adrenal hyperplasia. Psychoneuroendocrinology 1997; 22: $505-515$.

8. Hampson E, Rovet J, \& Altmann D. Spatial reasoning in children with congenital adrenal hyperplasia due to 21-hydroxylase deficiency. Dev Neuropsychol 1998; 14: 299-320.

9. Reiner W. Sex assignment in the neonate with intersex or inadequate genitalia. Arch Pediatr Adolesc Med 1997; 151(10):1044-5. 
10. Shaw A, Ardener S. Changing sex and bending gender, Berghahn Books 2005.

11. Money J. The concept of gender identity disorder in childhood and adolescence after 39 years. J Sex and Marital Ther 1994; 20(3): 163-77

12. Henslin, James M. Essentials of Sociology. Taylor \& Francis 2001; 240: 65-67.

13. Dessens, A.B., Slijper, F.M.E. and Drop, S.L.S.Gender dysphoria and gender change in chromosomal females with CAH. Arch Sex Behav 2005; 32(4): 389-397.

14. Mulaikal, R. M., Migeon, C. J., Rock, J. A. Fertility rates in female patients with congenital adrenal hyperplasia due to 21-hydroxylase deficiency. N Engl J Med 1987; 316: $178-182$.

15. Meyer-Bahlburg, H. F. L. What causes low rates of child-bearing in congenital adrenal hyperplasia? J Clin Endocrinol Metab 1999; 84: 1844-1847.

16. R. W. Dittmann, M. E. Kappes, and M. H. Kappes, Sexual behavior in adolescent and adult females with congenital adrenal hyperplasia, Psychoneuroendocrinology 1992; 17(2-3): 153-170. 
Figure 1-Steroidogenic pathway in adrenal cortex

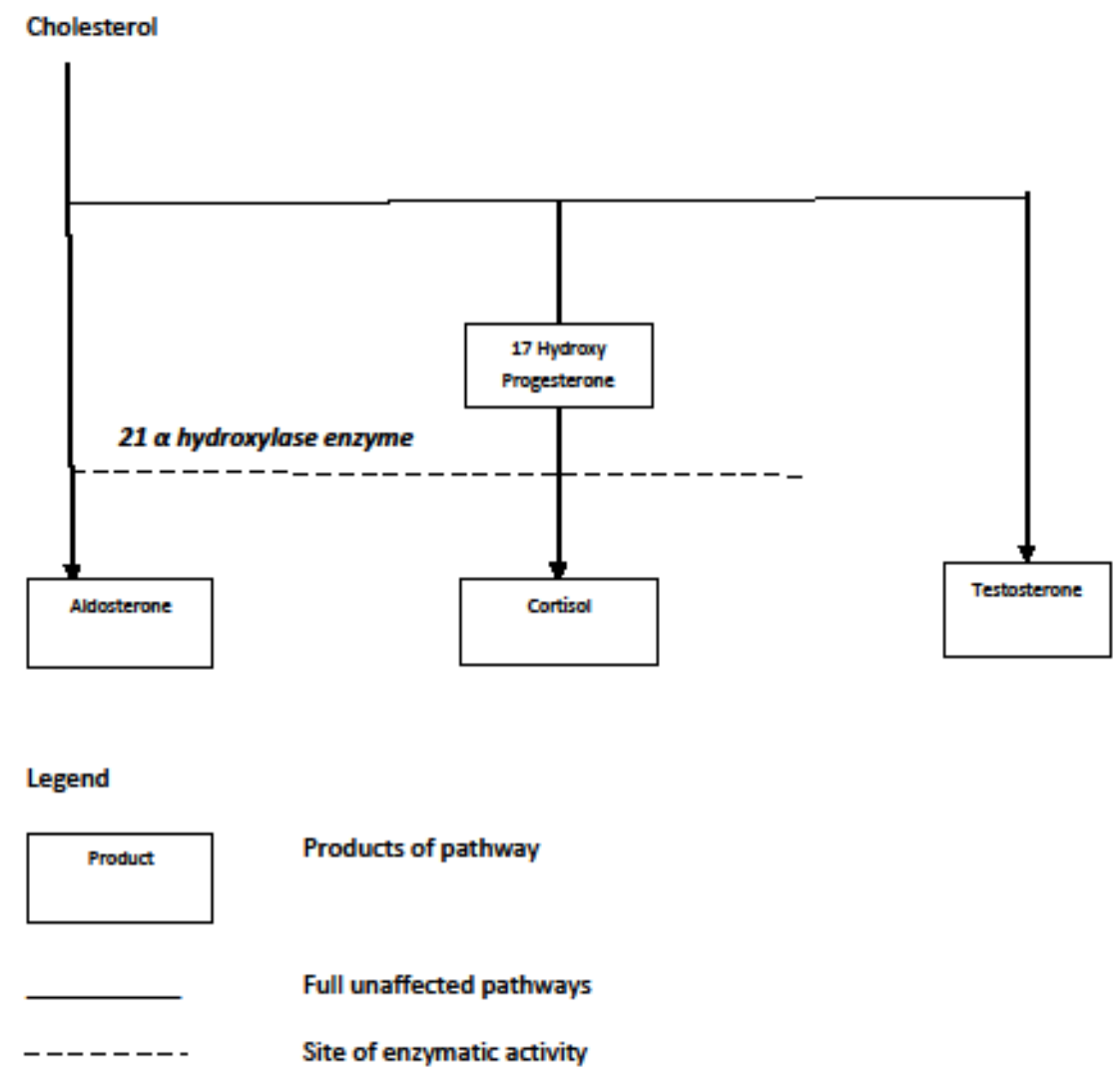

Figure: Steroidogenic pathway in adrenal cortext 